\title{
Progress and application on severe combined immunodeficiency mouse model for rheumatoid arthritis: a literature review
}

\author{
Zhipeng Li1 $\odot$, Ruifeng Yao' $\odot$, Yanqing Ying' ${ }^{\oplus}$, Junyu Qian ${ }^{1} \odot$,

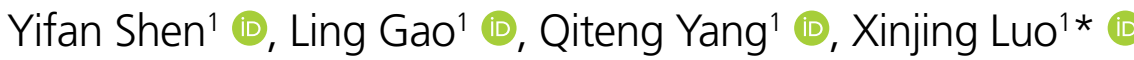

\section{INTRODUCTION}

Rheumatoid arthritis (RA) is a chronic progressive autoimmune disease $^{1}$ that can affect many tissues and organs, such as the heart and kidneys. Its pathogenesis is not completely stated. Previous studies ${ }^{2,3}$ have shown that it may be related to immune cells, autoantigens, and cytokines, and it is related to the intestinal flora. Some studies have reported that it may be related to genetic polymorphisms $s^{4,5}$. At present, the number of cases is increasing year by year, and the number of female patients is significantly higher than that of male patients ${ }^{6}$. If it is not treated or intervened in time, the disease has the risk of disability and teratogenesis, and in severe cases, it can endanger the life of the patient. To better understand the disease, it is particularly important to build good and easy-to-operate animal model for experimental. Pathology, immunology, and ethical regulations as well as technical limitations put forward higher requirements for animal models. Animals, such as mice, are good subjects for human disease models that summarize human symptoms and reactions. In addition, the genetic background of mice can be artificially altered to suit different situations ${ }^{7}$. Severe combined immunodeficiency (SCID) mice can provide an organizational environment that removes the effects of the immune system, and when incorporated into human RA synovial tissues, it is protected from the interference of the mouse immune system. Therefore, it is convenient for experimental research and quantitative analysis of the results.

\section{The process and methods for the} preparation of HuRAg-SCID mouse models The history of application in SCID mice dates back to the 1990s, when Geiler et al. ${ }^{8}$ implanted human RA synovial tissues and human normal cartilage into the kidney sacs of SCID mice for the first time. They also found that the transplanted synovial tissues survived well and was constantly growing. At the same time, they found that cartilage in contact with RA was significantly damaged and that the synovial tissues at the erosion site were more active in fibroblast proliferation. They successfully demonstrated that RA, in an environment away from human tissue, can grow in SCID mice and produce a large quantity of tissue factors and matrix degradation enzymes that erode cartilage. Consequently, the environment in SCID mice simulated the proliferation of human RA membrane cells, cartilage erosion, and degradation process. It also established a new humanized RA animal model. It is true that this method is free from the effects of the human tissue environment on RA erosion of cartilage. However, due to the limitation of scientific and technological research progress at that time, Geiler et al. ${ }^{8}$ did not transplant simple RA synovial tissue cells, but simply implanted a whole piece of synovial tissue. Although this method is simple to operate, it is inevitable that inflammatory factors and lymphocytes of local tissue in the human body will be transplanted into mice together. Therefore, the role of RASFs in cartilage erosion is not highlighted.

In response to the above problems, Lehmann et al. ${ }^{9}$ selectively isolated and cultured RASFs in vitro, purified, then directly injected them into the knee cavity of SCID mice. Finally, they found knee swelling, erosion, and destruction of articular cartilage surface, as well as a large number of Aschoff bodies in the synovial cavity. This method directly proves that RASFs' erosion of cartilage can be affected by human tissue environment and tissue factors. It provides a new direction for the follow-up

\footnotetext{
'Taizhou University, School of Medicine - Taizhou (Zhejiang), China.

*Corresponding author: luoxjing@126.com

Conflicts of interest: the authors declare there is no conflicts of interest. Funding: This project was funded by the Zhejiang Province Students Planted Talent Plan (2021R436020). The authors also thank the project organizing committee for the financial assistance provided to the author of this article. Received on July 29, 2021. Accepted on September 03, 2021.
} 
experiment. Lehmann et al. ${ }^{9}$ in vitro separation culture RASFs, injected into sterile gelatin sponge, and then the two with normal cartilage implanted in the kidney sacs of mice, found that RASFs in mice can still maintain the transformation and secretion activity, express the vascular cell adhesion molecule 1 (VCAM-1), and secrete cathepsin B and L to erode cartilage. On the basis of Lehmann et al. ${ }^{9}$, Pap et al. ${ }^{10}$ constructed three-dimensional cartilage-like matrix in vitro. This method of operation is to send RASFs, sterile gelatin sponge, and the body's normal synovial tissue in vitro suitable $\mathrm{pH}$ and ion concentration in suspension culture. Subsequently, Lefevre et al. ${ }^{11}$ innovatively transplanted the RASFs-cartilage-sterile sponge gelatin complex into one side of the mouse subcutaneously, and the other side was simultaneously transplanted with normal human articular cartilage. RASFs were injected subcutaneously, intraperitoneally, and intravenously into human mice. RASFs were detected in the blood and spleen of mice, which confirmed that RASFs migrated through the blood circulation in the body and could erode the cartilage of the contralateral or distant knee joint.

In recent years, scholars have continuously enriched and expanded the application of SCID mouse models in the field of RA research. Serrati et al. ${ }^{12}$ found that in vitro and SCID mouse models, the decrease in urokinase plasminogen activator receptor (UPAR) expression in RASFs reduced the damage of RASFs to cartilage and bone. Frey et al. ${ }^{13}$ isolated fibroblast-like synoviocytes (FLSs), expanded them in vitro, and transferred them to the knee joint cavity of SCID mice. They found that FLSs from AIA mice were able to transfer arthritis to recipient SCID mice. FLSs metastasis induces chronic arthritis and can find inflammatory cell collection and significant cartilage damage. They also observed in a single joint arthritis model that FLSs isolated from the contralateral non-arthritis joint have the potential to cause arthritis. FLSs metastasis induces chronic arthritis and can observe inflammatory cell collection and significant cartilage damage. They also observed arthritis potential in FLSs, which is separated from the opposite side of the non-arthritis joint, in the monoarthritis model. The transformation of these cells into arthritic cells occurs early in the development of arthritis. This challenges current assumptions about the role of FLSs in the pathogenesis of arthritis and opens the way for further mechanism research.

\section{Application of SCID-HuRAg mouse model}

\section{Application of the SCID-HuRAg model in virology and oncology and RA disease progression studies The pathogenesis of RA is extremely complex. As far as the current scientific research is concerned, its pathogenesis may be related to autoimmunity and digestive system. In recent}

years, there have been continuous reports in the scientific literature that the incidence of RA may also be related to viral infections and tumor attacks. Among them, the most extensive research is the relationship between Epstein-Barr virus (EBV) and RA and the functional changes or mutations of the tumor suppressor gene $\mathrm{p} 53$.

Many human viruses, including EBV, do not infect mice, which is challenging for biomedical research. The research team of Nagasawa et al. ${ }^{14}$ found that human osteoclasts mainly induce erosive arthritis during EBV infection. They cultivated bone marrow cells from EBV-infected SCID-HuRAg mice and analyzed their characteristics. Multinucleated cells cultured from bone marrow cells stained positive for human cathepsin $\mathrm{K}$ and human MMP-9, indicating that bone marrow cells of SCID-HuRAg mice could differentiate from human osteoclast progenitors into human osteoclasts, the human immune response to EBV infection may induce the activation of human osteoclasts and cause erosive arthritis in this mouse model. This study is also the first to demonstrate human osteoclast genesis in humanized mice. This model can be used to study the relationship between EBV infection and RA and human bone metabolism.

\section{Application of SCID-HuRAg model in pharmaceutical research and drug screening}

The special immune environment provided by SCID-HuRAg can highlight the erosion and degradation of cartilage and bone by RASFs. RASFs can secrete more cytokines, such as IL-1, IL-6, IL-18, and TNF- $\alpha^{15,16}$. Through these tissue factors, it plays its role in erosion and diffusion. In recent years, the focus of RA treatment programs has shifted from simply controlling pain and other characteristics to more effective antirheumatic treatments that control synovial hyperplasia and cartilage erosion and protect joint function. The SCID-HuRAg model has gradually begun to play an important role in pharmacological research and drug screening processes.

Therapies successfully tested in mice with a functional human immune system include the antiviral drug poly Pegylated Interferon Alpha-2a (Peg-IFN $\alpha 2 a)$, which shows signs of hepatitis $C$ virus suppression, such as human interferon- $\gamma$ production decrease, serum alanine aminotransferase level, hepatitis $\mathrm{C}$ virus ribonucleic acid copy number, and no leukocyte infiltration or fibrosis in the liver. Close to the clinical situation, humanized mice administered with ipilimumab developed autoimmune diseases, showing signs of weight loss, antinuclear antibodies, and increased adrenaline, which would aggravate the erosion and destruction effects of RASFs on cartilage and bone. In addition, theralizumab, a biological agent highly specific to human CD28, was tested in humanized mice implanted with 
polybrominated diphenyl ethers. These mice showed severe reduction in $\mathrm{CD} 45^{+}$human cells, rapid decline in body temperature, and elevated cytokine levels. They were given treatment $6 \mathrm{~h}$ after antibody administration. The adverse effects in the clinic can be observed, which will also exacerbate the negative effect of RASFs on the body. Taking into account the advantages, limitations, and potential development of humanized mice, the current data ${ }^{17}$ show that these models are useful tools for researchers to investigate short-term and long-term studies of therapeutic interactions and toxicity in vivo to reduce risks and ensure safety of healthy volunteers and patients exposed to drug candidates during clinical trials.

\section{CONCLUSIONS}

In recent years, SCID-HuRAg model mice have played an important role in the pathogenesis and pathological research of RA. At the same time, this model is also continuously applied to the research and development of new drugs, as well as pharmacodynamics and pharmacokinetics. Based on SCID-HuRAg model mice, human RA synovial cells or tissues combined with normal cartilage tissue or cells of the human body are embedded in mice in different ways, and the embedding positions are also different, it can be selected at the joints of mice or subcutaneously in mice. In the case of complete lack of B cells and $\mathrm{T}$ cells, study the changes of RASFs and the different responses of surrounding tissue factors and cytokines, explore the pathological process of cartilage and bone tissue erosion and destruction, and explore the possible pathogenesis of RA. The SCID-HuRAg model mouse highlights the relationship between RASFs and cartilage. At present, research on RA has gradually adopted this model and has begun to develop transgene therapy, RA, and other diseases on this model platform. It has an irreplaceable role in the treatment of cartilage erosion and degradation, as well as various body manifestations caused by inflammation. The application of this model mouse is to further improve and explore the pathogenesis of RA. With the deepening of research on the mechanism of RA disease, the quality of life of RA patients is expected to gradually improve.

\section{ACKNOWLEDGMENTS}

The authors thank colleagues in Taizhou University, Juan Zhang for the help with literature collection, and Tinghui Li, Chenchen Pan for proofreading the article.

\section{AUTHORS" CONTRIBUTIONS}

ZL: Conceptualization, Data curation, Formal analysis, Writing - original draft. RY: Data curation. YY: Data curation. JQ: Data curation. YS: Data curation, Writing - original draft. LG: Data curation, Formal analysis. QY: Data curation, Formal analysis. XI: Conceptualization, Writing - review \& editing.

\section{REFERENCES}

1. Zong D, Huang B, Li Y, Lu Y, Xiang N, Guo C, et al. Chromatin accessibility landscapes of immune cells in rheumatoid arthritis nominate monocytes in disease pathogenesis. BMC Biol. 2021;19(1):79. https://doi.org/10.1186/s12915-021-01011-6

2. Rana AK, Li Y, Dang Q, Yang F. Monocytes in rheumatoid arthritis: circulating precursors of macrophages and osteoclasts and, their heterogeneity and plasticity role in RA pathogenesis. Int Immunopharmacol. 2018;65:348-59. https://doi.org/10.1016/j. intimp.2018.10.016

3. Firestein GS, McInnes IB. Immunopathogenesis of rheumatoid arthritis. Immunity. 2017;46(2):183-96. https://doi. org/10.1016/j.immuni.2017.02.006

4. Tsai CY, Hsieh SC, Liu CW, Lu CH, Liao HT, Chen MH, et al. The expression of non-coding RNAs and their target molecules in rheumatoid arthritis: a molecular basis for rheumatoid pathogenesis and its potential clinical applications. Int J Mol Sci. 2021;22(11):5689. https://doi.org/10.3390/ijms22115689

5. Peng $Y$, Chen B, Sheng X, Qian Y. Polymorphisms in IRF5 and TYK2 genes are associated with rheumatoid arthritis in a Chinese han population. Med Sci Monitor. 2021;27:e928455. https://doi.org/10.12659/MSM.928455
6. Zhou C. A cross-sectional study of sexual dysfunction in chinese mainland female patients with rheumatoid arthritis. Arch Rheumatol. 2020;36(2):244-51. https://doi.org/10.46497/ ArchRheumatol.2021.8074

7. Xia X, Li H, Satheesan S, Zhou J, Rossi JJ. Humanized NOD/ SCID/IL2 rynull (hu-NSG) mouse model for HIV replication and latency studies. J Vis Exp. 2019(143):10.3791/58255. https:// doi.org/10.3791/58255

8. Geiler T, Kriegsmann J, Keyszer GM, Gay RE, Gay S. A new model for rheumatoid arthritis generated by engraftment of rheumatoid synovial tissue and normal human cartilage into SCID mice. Arthritis Rheum. 1994;37(11):1664-71. https://doi.org/10.1002/ art. 1780371116

9. Lehmann J, Jüngel $A$, Lehmann I, Busse $F$, Biskop $M$, Saalbach A, et al. Grafting of fibroblasts isolated from the synovial membrane of rheumatoid arthritis (RA) patients induces chronic arthritis in SCID mice-A novel model for studying the arthritogenic role of RA fibroblasts in vivo. J Autoimmun. 2000;15(3):301-13. https://doi.org/10.1006/ jaut. 2000.0435 
10. Pap $T$, van der Laan WH, Aupperle KR, Gay RE, Verheijen $\mathrm{JH}$, Firestein GS, et al. Modulation of fibroblast-mediated cartilage degradation by articular chondrocytes in rheumatoid arthritis. Arthritis Rheum. 2000;43(11):2531-6. https://doi.org/10.1002/15290131(200011)43:11<2531::AID-ANR21>3.0.CO;2-V

11. Lefèvre $S$, Knedla A, Tennie $C$, Kampmann A, Wunrau $C$, Dinser R, et al. Synovial fibroblasts spread rheumatoid arthritis to unaffected joints. Nat Med. 2009;15(12):1414-20. https:// doi.org/10.1038/nm.2050

12. Serratì $S$, Margheri $F$, Chillà $A$, Neumann E, Müller-Ladner $U$, Benucci $M$, et al. Reduction of in vitro invasion and in vivo cartilage degradation in a SCID mouse model by loss of function of the fibrinolytic system of rheumatoid arthritis synovial fibroblasts. Arthritis Rheum. 2011;63(9):2584-94. https://doi.org/10.1002/art.30439

13. Frey $\mathrm{O}$, Hückel $M$, Gajda $M$, Petrow PK, Bräuer R. Induction of chronic destructive arthritis in SCID mice by arthritogenic fibroblast-like synoviocytes derived from mice with antigeninduced arthritis. Arthritis Res Ther. 2018;20(1):261. https:// doi.org/10.1186/s13075-018-1720-y
14. Nagasawa $Y$, Takei M, Iwata M, Nagatsuka $Y$, Tsuzuki H, Imai K, et al. Human osteoclastogenesis in Epstein-Barr virus-induced erosive arthritis in humanized NOD/Shi-scid/L-2Rynull mice. PLoS One. 2021;16(4):e0249340. https://doi.org/10.1371/ journal.pone.0249340

15. Takeuchi T, Yoshida H, Tanaka S. Role of interleukin-6 in bone destruction and bone repair in rheumatoid arthritis. Autoimmun Rev. 2021;20(9):102884. https://doi.org/10.1016/j. autrev.2021.102884

16. Sung YK, Lee YH. Placebo and nocebo responses in randomized controlled trials of non-tumor necrosis factor biologics and Janus kinase inhibitors in patients with active rheumatoid arthritis showing insufficient response to tumor necrosis factor inhibitors: a meta-analysis. Z Rheumatol. 2021. https://doi. org/10.1007/s00393-021-01047-7

17. Yong KSM, Her Z, Chen Q. Humanized mice as unique tools for human-specific studies. Arch Immunol Ther Exp (Warsz). 2018;66(4):245-66. https://doi.org/10.1007/s00005-0180506-x 